\title{
Fractionation of the influenza virus with the object of producing subunit vaccine
}

\author{
L. HoYle \\ M.B., Ch.B.
}

\author{
SHEILA M. BARKER \\ B.Sc.
}

Public Health Laboratory, General Hospital, Northampton

\begin{abstract}
Summary
Attempts have been made to produce a subunit influenza vaccine containing haemagglutinin and neuraminidase but free of lipid and nucleic acid.

Disintegration of virus with Nonidet P40 followed by selective dialysis was only partially successful, but by disintegration with sodium dodecyl sulphate followed by ether extraction and precipitation with ammonium sulphate a product was obtained in which the content of ribonucleic acid was reduced to $1 \%$ and the lipid content to less than $5 \%$ of the amounts present in the original virus.
\end{abstract}

When A1 strains of virus and some strains of virus $A$ were shaken with $n$-butanol only the ribonucleoprotein (S antigen) was found in the aqueous phase and the procedure affords a very simple method of producing pure $S$ antigen for use in serological diagnosis.

\section{Introduction}

Influenza viruses are readily disintegrated into their component subunits by treatment with detergents or organic solvents but it is difficult to separate the subunits because on removal of the disintegrating agent the subunits tend to re-aggregate, especially if the preparations are of high concentration. During studies of this re-aggregation (Almeida \& Hoyle, 1972) observations were made which suggested the possibility of producing by simple methods a product which contained haemagglutinin and neuraminidase but little or no lipid or nucleic acid. Such a product might make an ideal subunit vaccine.

\section{Methods and results}

Three fractionation procedures have been studied. In each case the starting material was a virus preparation purified by two cycles of adsorptionelution from red blood cells followed by differential centrifugation. The final deposited virus was suspended in phosphate buffered saline to a concentration of about $1 \%$ by volume.

In most experiments in order to follow the distribution of lipid and nucleic acid during fractionation a small amount of virus labelled with ${ }^{32} \mathrm{P}$ was added to the stock virus.

\section{Disintegration with Nonidet P40}

A preparation of Hong Kong virus was mixed with an equal volume of $4 \%$ Nonidet $P 40$. This detergent splits the virus with the release of four major components, haemagglutinin, neuraminidase, ribonucleoprotein and lipoprotein. Separation of the components is difficult as removal of the detergent results in re-aggregation with the production of particles of mixed composition. Partial separation can be achieved by dialysis against water or buffers of low ionic strength.

On dialysis against $0.25 \%$ ammonium acetate a precipitate was formed which consisted mainly of haemagglutinin and neuraminidase, much of the ribonucleoprotein and most of the lipoprotein remaining in solution. The precipitate was centrifuged off and resuspended in distilled water and extracted with ether to remove Nonidet P40. On centrifugation the haemagglutinin and neuraminidase became highly aggregated at the ether-water interface in the form of separate clumps of very characteristic appearance in the electron microscope (Almeida \& Hoyle, 1972). On being suspended in buffered saline the material remained highly aggregated. It showed a high neuraminidase activity but because of the aggregation and high neuraminidase content it was not possible to determine directly the haemagglutinin content.

When the procedure was repeated using virus labelled with ${ }^{32} \mathrm{P}$ the final product was found to contain more lipid and RNA than had been expected. A typical final preparation was estimated to contain $76 \%$ of the original haemagglutinin and neuraminidase, $50 \%$ of the RNA and $10 \%$ of the original lipid. Improvement could only be achieved at the expense of a reduction in yield of the protein components.

\section{Disintegration with sodium dodecyl sulphate}

Sodium dodecyl sulphate is a more powerful detergent than Nonidet P40 and besides splitting virus into its component subunits it also splits RNA and lipid from combination with protein. Reaggregation of subunits only occurs after complete removal of SDS. 
To a mixture of $0.5 \mathrm{ml}$ of Hong Kong virus concentrate was added $0.3 \mathrm{ml}$ of ${ }^{32} \mathrm{P}$-labelled concentrate, $5.0 \mathrm{ml}$ of buffer pH 6.5 , and $0.8 \mathrm{ml}$ of $4 \%$ SDS. After $15 \mathrm{~min} 5.0 \mathrm{ml}$ of ether was added and the aqueous phase saturated with ammonium sulphate. On centrifugation the virus lipid was found in the ether layer, RNA remained in solution in the aqueous phase, while the virus proteins were precipitated as an interface layer. Ether and aqueous phases were removed and the interface material resuspended in saturated ammonium sulphate and again extracted with ether, and this procedure was repeated twice more. The final interface material was suspended in buffer and dialysed until free of ammonium sulphate. The original virus dilution gave a Geiger count of $1702 \mathrm{ct} / \mathrm{min} / \mathrm{ml}$ of which 872 represented alcohol soluble (lipid) ${ }^{32} \mathrm{P}$ and 830 was water-soluble RNA ${ }^{32} \mathrm{P}$. The final product gave a count of 48 $\mathrm{ct} / \mathrm{min} / \mathrm{ml}$ of which 42 was lipid and 6 RNA ${ }^{32} \mathrm{P}$. It therefore contained $4.8 \%$ of the original lipid and $0.7 \%$ of the original RNA.

Some mechanical losses of interface protein occurred during the processing but over a series of experiments mean recoveries of $90 \%$ were obtained. The material was highly aggregated and serological titres were low, but neuraminidase activity was high suggesting that the proteins were not seriously damaged.

\section{Disintegration with $\mathrm{n}$-butanol}

Purified virus concentrates were diluted $1: 10$ with buffer pH 6.5 and shaken with an equal volume of $n$-butanol. On centrifugation a layer of insoluble protein collected at the interface. Butanol and aqueous phases were removed and the interface material resuspended in buffer and cleared of butanol by three extractions with ether.

Results depended on the strain of virus used. In all cases the virus lipid was extracted by the butanol.
With $A_{1}$ strains and some strains of virus $A$ in which haemagglutinin and neuraminidase appear to be closely linked, both agents were precipitated and only the RNP (S antigen) remained in the aqueous phase. Serological and radioactive tracer studies showed that $90 \%$ of the total virus RNP antigen could be recovered. With $A_{2}$ strains in which haemagglutinin and neuraminidase are present as separate subunits most of the neuraminidase appeared in the aqueous phase along with the RNP antigen.

The interface material consisting mainly of haemagglutinin was highly aggregated and difficult to resuspend. Serological titres were low or negative and tracer studies indicated the presence of about $10 \%$ of the original RNA and lipid.

\section{Discussion}

The attempt to produce a subunit influenza vaccine containing haemagglutinin and neuraminidase but free from lipid and RNA has not been entirely successful, but significant reduction in lipid and RNA content without great loss of protein can be obtained and the methods are undergoing further study. The products are being tested for immunogenic potency and preliminary results indicate that they do have protective value.

The disintegration of $A_{1}$ strains of virus with $n-8$ butanol has proved of value as a very simple method of producing pure $\mathbf{S}$ antigen for use in serologicabe diagnosis.

I regret to announce that, since the writing of this paper, work on this project has been interrupted by the sudden death of Dr Hoyle. I should like to take this opportunity to express the degree of privilege I felt of working so closely with so eminent a virologist as Dr Hoyle. S.M.B.

\section{Reference}

Almeida, J.D. \& Hoyle, L. (1972) The reaggregation of influenza virus sub-units produced by detergent treatment of virus particles. Micron. In press. 